\title{
Probability Estimation in the Framework of Intuitionistic Fuzzy Evidence Theory
}

\author{
Yafei Song and Xiaodan Wang \\ Air and Missile Defense College, Air Force Engineering University, Xian 710051, China \\ Correspondence should be addressed to Yafei Song; yafei_song@163.com
}

Received 10 November 2014; Revised 24 March 2015; Accepted 9 April 2015

Academic Editor: Joao B. R. Do Val

Copyright ( 2015 Y. Song and X. Wang. This is an open access article distributed under the Creative Commons Attribution License, which permits unrestricted use, distribution, and reproduction in any medium, provided the original work is properly cited.

\begin{abstract}
Intuitionistic fuzzy (IF) evidence theory, as an extension of Dempster-Shafer theory of evidence to the intuitionistic fuzzy environment, is exploited to process imprecise and vague information. Since its inception, much interest has been concentrated on IF evidence theory. Many works on the belief functions in IF information systems have appeared. Although belief functions on the IF sets can deal with uncertainty and vagueness well, it is not convenient for decision making. This paper addresses the issue of probability estimation in the framework of IF evidence theory with the hope of making rational decision. Background knowledge about evidence theory, fuzzy set, and IF set is firstly reviewed, followed by introduction of IF evidence theory. Axiomatic properties of probability distribution are then proposed to assist our interpretation. Finally, probability estimations based on fuzzy and IF belief functions together with their proofs are presented. It is verified that the probability estimation method based on IF belief functions is also potentially applicable to classical evidence theory and fuzzy evidence theory. Moreover, IF belief functions can be combined in a convenient way once they are transformed to interval-valued possibilities.
\end{abstract}

\section{Introduction}

The Dempster-Shafer theory of evidence, also called belief function theory, is an important method to deal with uncertainty in information systems. Since it was firstly presented by Dempster [1], and was later extended and refined by Shafer [2], the Dempster-Shafer theory, or the D-S theory for short, has generated considerable interest. Its application has extended to many areas such as expert systems [3], fault reasoning $[4,5]$, pattern classification [6-9], information fusion [10], knowledge reduction [11], global positioning system [12], regression analysis [13], and data mining [14].

The theory of fuzzy set, proposed by Zadeh [15], is another mathematical tool for handling uncertainty. It has received a great deal of attention due to its capability in uncertainty reasoning. Therefore, over the last decades, several generalizations of fuzzy set have been introduced in the literature. Intuitionistic fuzzy (IF) set proposed by Atanassov [16] is one of the generalizations of fuzzy set which is capable of dealing with vagueness much better. A fuzzy set only gives a membership degree to describe an element belonging to a set, while an intuitionistic fuzzy set gives both a membership degree and a nonmembership degree. Thus, an IF set is more objective than a fuzzy set to describe the vagueness of data. As a fuzzy set can be reviewed as a fuzzy event, an IF set is also an IF event.

Relationship between fuzzy set theory and belief function theory has been focused on for a long time. Zadeh was the first to generalize the Dempster-Shafer theory to fuzzy sets, based on his work on the concept of information granularity and the theory of possibility $[17,18]$. He suggested how to compute probabilities of fuzzy events and showed some basic properties of probabilities of fuzzy events [19]. Following Zadeh's work, Ishizuka, Yager, Ogawa, John Yen, and Zhu have extended the D-S theory to fuzzy sets in different ways [20-24]. As an inception, belief functions on IF events were investigated by Grzegorzewski and Mrówka in [25], where basic properties of probability measures for IF events were studied. Riečan gave an axiomatic characterization of a probability on IF events in [26] and proved a representation theorem for it in [27]. In [28, 29], Gerstenkorn and Mańko gave two new definitions of the IF probability: the first 
probability of an IF set is a real number in $[0,1]$ using the integral operation, and the second probability of an IF set is also an IF set based on the level sets. In [30], Gerstenkorn and Mańko defined a probability of IF events, which is defined by the membership degree and half of the hesitancy margin of every element, where the probability of an IF set is a real number. Feng et al. [31] proposed a novel pair of belief and plausibility functions defined by employing intuitionistic fuzzy lower and upper approximation operators.

All the above works on IF belief function theory focused on the determination of the basic probabilities assigned to IF events based on the known probabilistic distribution in the universe of discourse. However, its converse problem, that is, probability estimate through belief functions on IF events, is rarely addressed. Although belief functions on IF events can handle uncertainty better, it is not a good tool for decision making. Therefore, a transformation that can transform belief functions on IF events to probability distribution in the universe is desirable for the sake of sounder decision.

In this paper, we investigate the probability estimation of belief functions on IF event to cope with the issue of decision making in the framework of IF evidence theory. A method to estimate the probability distribution in the universe of discourse is proposed in this paper. Since the probability of each basic event tends to be defined as an interval value on the unit interval $[0,1]$, decision making will be implemented based on interval comparison. Moreover, it is easy to verify that this transformation will reduce to the pignistic transformation when the focal elements are crisp sets. As for singleton focal elements, the concept of basic probability assignment (BPA), belief function, and the probability of IF events are identical. So we will adopt the terminology of BPA and belief function for IF events instead of the probability of IF events, which has appeared in other literatures. Besides, the terminology of probability is used to express the probability distribution on the basic events in the universe of discourse.

The remainder of this paper is organized as follows. Section 2 gives a brief recall of the $\mathrm{D}$-S theory of evidence and IF sets, as well as IF evidence theory. In Section 3, we present axiomatic requirements for probability distribution, followed by the definition of probability estimation based on IF belief functions. Illustrative examples are presented in Section 4 to show the applicability of our proposed method. In Section 5, we derive the conclusion of this paper.

\section{Background}

The background material presented in this section deals with the following three main points: (1) the interpretation of Dempster-Shafer theory of evidence, which will be used in this paper to ease the exposition, (2) a brief review of definitions on fuzzy set and IF set, and (3) introduction of intuitionistic fuzzy evidence theory.

2.1. Dempster-Shafer Theory of Evidence. Dempster-Shafer theory of evidence was modeled based on a finite set of mutually exclusive elements, called the frame of discernment denoted by $\Omega$ [1]. The power set of $\Omega$, denoted by $2^{\Omega}$, contains all possible unions of the sets in $\Omega$. Singleton sets in a frame of discernment $\Omega$ will be called atomic sets because they do not contain nonempty subsets. The following definition is central in the Dempster-Shafer theory.

Definition 1. Let $\Omega=\left\{A_{1}, A_{2}, \ldots, A_{n}\right\}$ be the frame of discernment. A basic probability assignment (BPA) is a function $m: 2^{\Omega} \rightarrow[0,1]$, satisfying the two following conditions:

$$
\begin{aligned}
m(\varnothing) & =0 \\
\sum_{A \subseteq \Omega} m(A) & =1
\end{aligned}
$$

where $\varnothing$ denotes empty set and $A$ is any subset of $\Omega$. Such a function is also called a basic belief assignment by Smets [32] and a belief structure by Yager [33]. The terminology of belief function will be adopted in this paper. For each subset $A \subseteq \Omega$, the value taken by the BPA at $A$ is called the basic probability assigned to $A$, or the BPA of $A$ for short, denoted by $m(A)$.

Definition 2. A subset $A$ of $\Omega$ is called the focal element of a belief function $m$ if $m(A)>0$.

Definition 3. For a belief function $m$ on $\Omega$, the belief function and plausibility function which are in one-to-one correspondence with $m$ can be defined, respectively, as

$$
\begin{aligned}
\operatorname{Bel}(A) & =\sum_{B \subseteq A} m(B), \\
\operatorname{Pl}(A) & =\sum_{B \cap A \neq \varnothing} m(B)=1-\sum_{B \cap A=\varnothing} m(B) .
\end{aligned}
$$

Definition 4 (see $[32,34]$ ). The pignistic transformation maps a belief function $m$ to so-called pignistic probability function. The pignistic transformation of a belief function $m$ on $\Omega=$ $\left\{A_{1}, A_{2}, \ldots, A_{n}\right\}$ is given by

$$
\operatorname{BetP}(A)=\sum_{B \subseteq \Omega} \frac{|A \cap B|}{|B|} \frac{m(B)}{1-m(\varnothing)}, \quad \forall A \subseteq \Omega,
$$

where $|A|$ is the cardinality of set $A$.

Particularly, when $m(\varnothing)=0$ and $A \in \Omega$, that is, $A$ is a singleton set of $\Omega$, we have

$$
\operatorname{BetP}(A)=\sum_{A \in B} \frac{m(B)}{|B|}, \quad A=A_{1}, \ldots, A_{n}, B \subseteq \Omega .
$$

Definition 5. Given two belief functions $m_{1}$ and $m_{2}$ on $\Omega$, the belief function that results from the application of Dempster's combination rule, denoted as $m_{1} \oplus m_{2}$, or $m_{12}$ for short, is given by

$$
\begin{aligned}
m_{1} & \oplus m_{2}(A) \\
= & \begin{cases}\frac{\sum_{B \cap C=A} m_{1}(B) m_{2}(C)}{1-\sum_{B \cap C=\varnothing} m_{1}(B) m_{2}(C)}, & A \neq \varnothing, \\
0, & A=\emptyset .\end{cases}
\end{aligned}
$$


2.2. Intuitionistic Fuzzy Sets. In this section, we briefly recall the basic concepts related to fuzzy sets and intuitionistic fuzzy sets.

Definition 6 (see [15]). Let $X=\left\{x_{1}, x_{2}, \ldots, x_{n}\right\}$ be a universe of discourse; then a fuzzy set $A$ in $X$ is defined as

$$
A=\left\{\left\langle x, \mu_{A}(x)\right\rangle \mid x \in X\right\},
$$

where $\mu_{A}: X \rightarrow[0,1]$ is the membership degree.

Definition 7 (see [16]). An IF set $A$ in $X$ defined by Atanassov can be written as

$$
A=\left\{\left\langle x, \mu_{A}(x), v_{A}(x)\right\rangle \mid x \in X\right\}
$$

where $\mu_{A}: X \rightarrow[0,1]$ and are membership degree and nonmembership degree, respectively, with the condition:

$$
0 \leq \mu_{A}(x)+v_{A}(x) \leq 1 .
$$

The hesitancy degree $\pi_{A}(x)$ is determined by

$$
\pi_{A}(x)=1-\mu_{A}(x)-v_{A}(x) .
$$

$\pi_{A}(x)$ is also called the intuitionistic index of $x$ to $A$. Greater $\pi_{A}(x)$ indicates more vagueness on $x$. Obviously, when $\pi_{A}(x)=0, \forall x \in X$, the IF set degenerates into an ordinary fuzzy set. In the sequel, the couple $\left\langle\mu_{A}(x), v_{A}(x)\right\rangle$ is called an IF set or IF event for clarity. $\operatorname{IFS}(X)$ denotes the family of all IF sets in $X$.

Definition 8 (see [35]). For $A \in \operatorname{IFS}(X)$ and $B \in \operatorname{IFS}(X)$, some operations on them are defined as follows:

(1) $A \subset B$ iff, $\forall x \in X \mu_{A}(x) \leq \mu_{B}(x), v_{A}(x) \geq v_{B}(x)$.

(2) $A=B$ iff, $\forall x \in X \mu_{A}(x)=\mu_{B}(x), v_{A}(x)=v_{B}(x)$.

(3) $A^{C}=\left\{\left\langle x, v_{A}(x), \mu_{A}(x)\right\rangle \mid x \in X\right\}$, where $A^{C}$ is the complementary set of $A$.

It is worth noting that, besides Definition 7, there are other possible representations of IF sets proposed in the literature. Hong and Choi [36] proposed to use an interval representation $\left[\mu_{A}(x), 1-v_{A}(x)\right]$ of intuitionistic fuzzy set in $X$ instead of the couple $\left\langle\mu_{A}(x), v_{A}(x)\right\rangle$. This interpretation of IF set is equivalent to the interval-valued fuzzy sets, where the interval $\left[\mu_{A}(x), 1-v_{A}(x)\right]$ represents the membership degree of $x \in X$ to the set $A$. Obviously, $\left[\mu_{A}(x), 1-v_{A}(x)\right]$ is a valid interval, since $\mu_{A}(x) \leq 1-v_{A}(x)$ always holds for $\mu_{A}(x)+v_{A}(x) \leq 1$.

2.3. Intuitionistic Fuzzy Evidence Theory. It has been well known that Dempster-Shafer evidence theory can express and deal with uncertainty in crisp sets. However, D-S theory itself can not represent and manage vague information such as "the price is high" or "his age is about 40." To overcome this problem, fuzzy evidence theory was proposed to process imprecise and vague information [17-23].
Definition 9 (see [24]). Let $X=\left\{x_{1}, x_{2}, \ldots, x_{n}\right\}$ be a universe of discourse, and let FS $(X)$ be the family of all fuzzy sets in $X$. A fuzzy belief function $m$ is defined as

$$
\left\{\left\langle A_{i}^{F}, m\left(A_{i}^{F}\right), \mu_{A_{i}^{F}}\left(x_{j}\right)\right\rangle\right\}, \quad A_{i}^{F} \in \mathrm{FS}(X), x_{j} \in X,
$$

where $A_{i}^{F}$ is a normal fuzzy set, $\mu_{A_{i}^{F}}\left(x_{j}\right)$ is the membership function of the fuzzy set $A_{i}^{F}$, and $m\left(A_{i}^{F}\right)$ is the BPA of $A_{i}^{F}$. A fuzzy set $A^{F} \in \mathrm{FS}(X)$ with $m\left(A^{F}\right)>0$ is referred to as a focal element of $m$.

Definition 10 (see [17]). Let the probability of each element in $X$ be $P\left(x_{j}\right)(j=1,2, \ldots, n)$. The BPA of a fuzzy event $A_{i}^{F}=$ $\left\{\left\langle x_{j}, \mu_{A_{i}^{F}}\left(x_{j}\right)\right\rangle \mid x_{j} \in X\right\}$ is defined as

$$
m\left(A_{i}^{F}\right)=\sum_{j=1}^{n} P\left(x_{j}\right) \mu_{A_{i}^{F}}\left(x_{j}\right) .
$$

Definition 11. Let $X=\left\{x_{1}, x_{2}, \ldots, x_{n}\right\}$ be a universe of discourse, and let IFS $(X)$ be the family of all fuzzy sets in $X$. A fuzzy belief function $m$ is defined as

$$
\begin{aligned}
\left\{\left\langle A_{i}^{\mathrm{IF}}, m\left(A_{i}^{\mathrm{IF}}\right), \mu_{A_{i}^{\mathrm{IF}}}\left(x_{j}\right), v_{A_{i}^{\mathrm{IF}}}\left(x_{j}\right)\right\rangle\right\}, & \\
A_{i}^{\mathrm{IF}} & \in \operatorname{IFS}(X), x_{j} \in X,
\end{aligned}
$$

where $A_{i}^{\mathrm{IF}}$ is an intuitionistic fuzzy set, $\mu_{A_{i}^{\mathrm{IF}}}\left(x_{j}\right)$ is the membership function of the IF set $A_{i}^{\mathrm{IF}}, v_{A_{i}^{\mathrm{IF}}}\left(x_{j}\right)$ is the membership function, and $m\left(A_{i}^{\mathrm{IF}}\right)$ is the BPA of $A_{i}^{\mathrm{IF}}$.

Definition 12 (see [25]). Let the probability of each element in $X$ be $P\left(x_{j}\right)(j=1,2, \ldots, n)$. The BPA of an IF event $A_{i}^{\mathrm{IF}}=$ $\left\{\left\langle x_{j}, \mu_{A_{i}^{\mathrm{IF}}}\left(x_{j}\right), v_{A_{i}^{\mathrm{IF}}}\left(x_{j}\right)\right\rangle \mid x_{j} \in X\right\}$ is an interval value defined as

$$
m\left(A_{i}^{\mathrm{IF}}\right)=\left[m_{\min }\left(A_{i}^{\mathrm{IF}}\right), m_{\max }\left(A_{i}^{\mathrm{IF}}\right)\right]
$$

where $m_{\min }\left(A_{i}^{\mathrm{IF}}\right)$ and $m_{\max }\left(A_{i}^{\mathrm{IF}}\right)$ are the minimal and maximal BPA of $A_{i}^{\mathrm{IF}}$, respectively, defined as

$$
\begin{aligned}
m_{\min }\left(A_{i}^{\mathrm{IF}}\right) & =\sum_{j=1}^{n} P\left(x_{j}\right) \mu_{A_{i}^{\mathrm{IF}}}\left(x_{j}\right), \\
m_{\max }\left(A_{i}^{\mathrm{IF}}\right) & =\sum_{j=1}^{n} P\left(x_{j}\right)\left(1-v_{A_{i}^{\mathrm{IF}}}\left(x_{j}\right)\right) \\
& =m_{\min }\left(A_{i}^{\mathrm{IF}}\right)+\sum_{j=1}^{n} P\left(x_{j}\right) \pi_{A_{i}^{\mathrm{IF}}}\left(x_{j}\right) .
\end{aligned}
$$

It is easy to verify that $0 \leq m_{\min }\left(A_{i}^{\mathrm{IF}}\right) \leq m_{\max }\left(A_{i}^{\mathrm{IF}}\right) \leq$ 1. We will take the example analyzed in [37] to show the computation of BPA for IF events.

Example 13. Suppose there is a discussion on the amount of money which should be assigned for advertisement of a new 
product. Let us assume that the IF event $A$ corresponding to the statement "about fifty thousands" is given by

$$
\begin{aligned}
A= & \{\langle 30,0.2,0.6\rangle,\langle 40,0.5,0.4\rangle,\langle 50,1,0\rangle, \\
& \langle 60,0.5,0.4\rangle,\langle 70,0.2,0.6\rangle\} .
\end{aligned}
$$

The probability distribution of the amount of money is

$$
P(30)=P(40)=P(50)=P(60)=P(70)=0.2 \text {. }
$$

From (14) we get immediately

$$
\begin{aligned}
& m_{\text {min }}=0.2(0.2+0.5+1+0.5+0.2)=0.48, \\
& m_{\text {max }}=0.2(0.4+0.6+1+0.6+0.4)=0.6 .
\end{aligned}
$$

It means that the basic probability assigned to the event of "assigning about fifty thousands for advertisement" lies in the interval $[0.48,0.6]$.

Definition 14. Let $\mathbb{F}$ denote the set of all focal elements (including crisp sets, fuzzy sets, or IF sets), and let $|\mathbb{F}|$ denote the cardinality of $\mathbb{F}$. A belief function $m$ is a normalized belief function if it satisfies

$$
\sum_{A_{i} \in \mathbb{F}} m\left(A_{i}\right)=1
$$

The normalization of classical belief functions has been investigated in many works, for example, [38]. For nonnormalized fuzzy or intuitionistic fuzzy belief functions, the problem of their proper normalization arises. Normalization of fuzzy evidence has been proposed in [39] and is beyond the scope of this paper.

It is apparent that the proposed BPA in (13) reduces to Zadeh's probability measure in (11) when $A$ is a normal fuzzy set. We must note that there are many other methods for computing probability of fuzzy events and IF events. More recent results can be found in $[31,37]$.

\section{Probability Estimation Based on IF Belief Functions}

The advantage of belief function theory in IF set lies not only in its ability to express degree of uncertainty (nonspecificity, discord, and fuzziness), but also in its capacity for facilitating the fusion of uncertain information. However, since decision making based on classical belief function is still under dispute, how to make decision based on IF belief function is also worth studying.

In many practical information systems, the final outputs may be expressed by belief functions on IF events such as "about fifty thousands," which are given by experts without the probability distribution of basic events, or from the fusion result of several sensors with limit knowledge. Due to the fuzziness of IF events, few precise decisions can be made based on the probabilities assigned to them. In contrast, probability distribution on the specific events in the universe is more helpful for decision making. Therefore, the problem of probability estimation based on IF evidence arises. This issue can be illustrated by the following example.
Example 15. For the situation discussed in Example 13, let the universe of discourse be $X=\{30,40,50,60,70\}$. The assessment result from some experts is that the probability of "assigning about fifty thousands for advertisement" is 0.5 , the probability of "assigning a large amount of money for advertisement" is 0.2 , and the probability of "assigning a small amount of money for advertisement" is 0.3 . The statements "about fifty thousands," "a large amount of money," and "a small amount of money" can be expressed by three IF events $A, B$, and $C$, respectively, given by

$$
\begin{aligned}
A= & \{\langle 30,0.2,0.6\rangle,\langle 40,0.5,0.4\rangle,\langle 50,1,0\rangle, \\
& \langle 60,0.5,0.4\rangle,\langle 70,0.2,0.6\rangle\}, \\
B= & \{\langle 30,0,1\rangle,\langle 40,0.2,0.6\rangle,\langle 50,0.5,0.3\rangle, \\
& \langle 60,0.8,0.1\rangle,\langle 70,1,0\rangle\}, \\
C= & \{\langle 30,1,0\rangle,\langle 40,0.8,0.1\rangle,\langle 50,0.3,0.4\rangle, \\
& \langle 60,0.1,0.8\rangle,\langle 70,0,1\rangle\} .
\end{aligned}
$$

Experts' assessment result can be written as

$$
\begin{aligned}
& m(A)=0.5 \\
& m(B)=0.2 \\
& m(C)=0.3
\end{aligned}
$$

This assessment result is too approximate to leading to a precise decision for the decision maker. It can merely provide qualitative rather than quantitative guidance. Since the money assigned for the advertisement is a specific number in implement, such a result must be transformed to a probability distribution in the universe of discourse, which can also be regarded as a course of defuzzification in the IF belief function.

Definition 16. Let $X=\left\{x_{1}, x_{2}, \ldots, x_{n}\right\}$ be a universe of discourse. A mapping $P: X \rightarrow[0,1], P\left(x_{j}\right)(j=1,2, \ldots, n)$ is said to be a probability distribution in $X$ if $P\left(x_{j}\right)$ satisfies the following properties:
(1) $0 \leq P\left(x_{j}\right) \leq 1, \forall x_{j} \in X$.
(2) $\sum_{j=1}^{n} P\left(x_{j}\right)=1$.

Definition 17. Let $X=\left\{x_{1}, x_{2}, \ldots, x_{n}\right\}$ be a universe of discourse. $P\left(x_{j}\right)=\left[a_{j}, b_{j}\right]$ is the interval probability of $x_{j}$ with $0 \leq a_{j} \leq b_{j} \leq 1, j=1,2, \ldots, n . P\left(x_{j}\right)$ is said to be an interval-valued probability distribution in $X$ if $P\left(x_{j}\right)$ satisfies the following properties:

$$
\begin{aligned}
& \text { (1) } \sum_{j=1}^{n} b_{j}-\left(b_{k}-a_{k}\right) \geq 1, \forall k \in\{1, \ldots, n\} . \\
& \text { (2) } \sum_{j=1}^{n} a_{j}+\left(b_{k}-a_{k}\right) \leq 1, \forall k \in\{1, \ldots, n\} .
\end{aligned}
$$

Definition 18. Let $X=\left\{x_{1}, x_{2}, \ldots, x_{n}\right\}$ be a universe of discourse, and let $\mathbb{F}$ be the set of all focal elements. A normalized fuzzy belief function $m$ is given as

$$
\left\{\left\langle A_{i}^{F}, m\left(A_{i}^{F}\right), \mu_{A_{i}^{F}}\left(x_{j}\right)\right\rangle\right\}, \quad A_{i}^{F} \in \mathbb{F}, x_{j} \in X .
$$


Then, the probability of $x_{j}(j=1,2, \ldots, n)$ can be estimated as

$$
\widetilde{P}\left(x_{j}\right)=\sum_{A_{i}^{F} \in \mathbb{F}} \frac{m\left(A_{i}^{F}\right) \mu_{A_{i}^{F}}\left(x_{j}\right)}{\sum_{j=1}^{n} \mu_{A_{i}^{F}}\left(x_{j}\right)} .
$$

Proposition 19. The probability estimation $\widetilde{P}\left(x_{j}\right) \quad(j=$ $1,2, \ldots, n)$ is a probability distribution in $X=\left\{x_{1}, x_{2}, \ldots, x_{n}\right\}$.

Proof. Given $0 \leq m\left(A_{i}^{F}\right) \leq 1$, and $\sum_{A_{i}^{F} \in \mathbb{F}} m\left(A_{i}^{F}\right)=1$, we have

$$
\begin{aligned}
\widetilde{P}\left(x_{j}\right) & =\sum_{A_{i}^{F} \in \mathbb{F}} \frac{m\left(A_{i}^{F}\right) \mu_{A_{i}^{F}}\left(x_{j}\right)}{\sum_{j=1}^{n} \mu_{A_{i}^{F}}\left(x_{j}\right)} \geq 0, \\
\widetilde{P}\left(x_{j}\right) & =\sum_{A_{i}^{F} \in \mathbb{F}} \frac{m\left(A_{i}^{F}\right) \mu_{A_{i}^{F}}\left(x_{j}\right)}{\sum_{j=1}^{n} \mu_{A_{i}^{F}}\left(x_{j}\right)} \leq \sum_{A_{i}^{F} \in \mathbb{F}} m\left(A_{i}^{F}\right)=1, \\
\sum_{j=1}^{n} \widetilde{P}\left(x_{j}\right) & =\sum_{j=1}^{n} \sum_{A_{i}^{F} \in \mathbb{F}} \frac{m\left(A_{i}^{F}\right) \mu_{A_{i}^{F}}\left(x_{j}\right)}{\sum_{j=1}^{n} \mu_{A_{i}^{F}}\left(x_{j}\right)} \\
& =\sum_{A_{i}^{F} \in \mathbb{F} j=1} \sum_{j=1}^{n} \frac{m\left(A_{i}^{F}\right) \mu_{A_{i}^{F}}\left(x_{j}\right)}{\sum_{j=1}^{n} \mu_{A_{i}^{F}}\left(x_{j}\right)} \\
& =\sum_{A_{i}^{F} \in \mathbb{F}} m\left(A_{i}\right)\left(\sum_{j=1}^{n} \frac{\mu_{A_{i}^{F}}\left(x_{j}\right)}{\sum_{j=1}^{n} \mu_{A_{i}^{F}}\left(x_{j}\right)}\right) \\
& =\sum_{A_{i} \in \mathbb{F}} m\left(A_{i}\right)=1 .
\end{aligned}
$$

Therefore, $\widetilde{P}\left(x_{j}\right)$ is a probability distribution in $X$.

The multielement focal elements for classical belief function can be also regarded as fuzzy sets, where the membership degrees of elements take values 0 or 1 . For example, the focal element $A=\left\{x_{1}, x_{2}, x_{4}\right\}$ in the universe $X=\left\{x_{1}, x_{2}, x_{3}, x_{4}, x_{5}\right\}$ can be written as a fuzzy set as $A^{F}=\left\{\left\langle x_{1}, 1\right\rangle,\left\langle x_{2}, 1\right\rangle,\left\langle x_{3}, 0\right\rangle,\left\langle x_{4}, 1\right\rangle,\left\langle x_{5}, 0\right\rangle\right\}$. Given $m(A)=$ $m\left(A^{F}\right)$, we can get $\operatorname{BetP}\left(x_{1}\right)=\operatorname{BetP}\left(x_{2}\right)=\operatorname{BetP}\left(x_{4}\right)=$ $m(A) / 3$ and $\widetilde{P}\left(x_{1}\right)=\widetilde{P}\left(x_{2}\right)=\widetilde{P}\left(x_{4}\right)=m\left(A^{F}\right) / 3$ from the pignistic transformation in (4) and the probability estimation in (22), respectively. In such case, the probability estimation $\widetilde{P}\left(x_{j}\right)$ coincides with the pignistic probability. So the probability estimation based on fuzzy belief functions can be regarded as a generalization of the pignistic transformation.

Since a probability distribution generates interval-valued belief functions for IF events, the probability estimation through IF belief functions should also be interval values. Therefore, for each $x_{j} \in X$, the minimal probability $P_{\min }\left(x_{j}\right)$ and the maximal probability $P_{\max }\left(x_{j}\right)$ give the lowest and the highest possible probability that the event $x_{j}$ will occur, respectively.
Definition 20. Let $X=\left\{x_{1}, x_{2}, \ldots, x_{n}\right\}$ be a universe of discourse, and let $\mathbb{F}$ be the set of all focal elements. An intuitionistic fuzzy belief function $m$ is given as

$$
\begin{aligned}
\left\{\left\langle A_{i}^{\mathrm{IF}}, m\left(A_{i}^{\mathrm{IF}}\right), \mu_{A_{i}^{\mathrm{IF}}}\left(x_{j}\right), v_{A_{i}^{\mathrm{IF}}}\left(x_{j}\right)\right\rangle\right\}, & \\
& A_{i}^{\mathrm{IF}} \in \mathbb{F}, x_{j} \in X .
\end{aligned}
$$

Then, the probability of $x_{j}, j=1,2, \ldots, n$, can be estimated as

$$
\widetilde{P}\left(x_{j}\right)=\left[\widetilde{a}_{j}, \widetilde{b}_{j}\right],
$$

where $\widetilde{a}_{j}$ and $\widetilde{b}_{j}$ are defined as

$$
\begin{aligned}
& \tilde{a}_{j}=\sum_{A_{i}^{\mathrm{IF}} \in \mathbb{F}} \frac{m\left(A_{i}^{\mathrm{IF}}\right) \mu_{A_{i}^{\mathrm{IF}}}\left(x_{j}\right)}{\sum_{j=1}^{n}\left(1-v_{A_{i}^{\mathrm{IF}}}\left(x_{j}\right)\right)-\pi_{A_{i}^{\mathrm{IF}}}\left(x_{j}\right)}, \\
& \widetilde{b}_{j}=\sum_{A_{i}^{\mathrm{IF}} \in \mathbb{E}} \frac{m\left(A_{i}^{\mathrm{IF}}\right)\left(1-v_{A_{i}^{\mathrm{IF}}}\left(x_{j}\right)\right)}{\sum_{j=1}^{n} \mu_{A_{i}^{\mathrm{IF}}}\left(x_{j}\right)+\pi_{A_{i}^{\mathrm{IF}}}\left(x_{j}\right)} .
\end{aligned}
$$

Proposition 21. The interval probability estimation $\widetilde{P}\left(x_{j}\right)=$ $\left[\widetilde{a}_{j}, \widetilde{b}_{j}\right](j=1,2, \ldots, n)$ is an interval-valued probability distribution in $X=\left\{x_{1}, x_{2}, \ldots, x_{n}\right\}$.

Proof. The relationships between $\mu_{A_{i}^{\mathrm{IF}}}\left(x_{j}\right), v_{A_{i}^{\mathrm{IF}}}\left(x_{j}\right)$, and $\pi_{A_{i}^{\mathrm{IF}}}\left(x_{j}\right)$ are as follows:

$$
\begin{aligned}
\mu_{A_{i}^{\mathrm{IF}}}\left(x_{j}\right)+v_{A_{i}^{\mathrm{IF}}}\left(x_{j}\right) & \leq 1, \\
\mu_{A_{i}^{\mathrm{IF}}} & \left(x_{j}\right) \leq 1-v_{A_{i}^{\mathrm{IF}}}\left(x_{j}\right), \\
\pi_{A_{i}^{\mathrm{II}}}\left(x_{j}\right) & =1-\mu_{A_{i}^{\mathrm{IF}}}\left(x_{j}\right)-v_{A_{i}^{\mathrm{II}}}\left(x_{j}\right) .
\end{aligned}
$$

Form the above relationships we can get

$\widetilde{a}_{j}$

$$
\begin{aligned}
& =\sum_{A_{i}^{\mathrm{IF}} \in \mathbb{F}} \frac{m\left(A_{i}^{\mathrm{IF}}\right) \mu_{A_{i}^{\mathrm{IF}}}\left(x_{j}\right)}{\sum_{j=1}^{n}\left(1-v_{A_{i}^{\mathrm{IF}}}\left(x_{j}\right)\right)-\pi_{A_{i}^{\mathrm{IF}}}\left(x_{j}\right)} \\
& =\sum_{A_{i}^{\mathrm{I}} \in \mathbb{F}} \frac{m\left(A_{i}^{\mathrm{IF}}\right) \mu_{A_{i}^{\mathrm{IF}}}\left(x_{j}\right)}{\sum_{k=1, k \neq j}^{n}\left(1-v_{A_{i}^{\mathrm{II}}}\left(x_{k}\right)\right)+1-v_{A_{i}^{\mathrm{IF}}}\left(x_{j}\right)-\pi_{A_{i}^{\mathrm{II}}}\left(x_{j}\right)} \\
& =\sum_{A_{i}^{\mathrm{I}} \in \mathbb{E}} \frac{m\left(A_{i}^{\mathrm{IF}}\right) \mu_{A_{i}^{\mathrm{IF}}}\left(x_{j}\right)}{\sum_{k=1, k \neq j}^{n}\left(1-v_{A_{i}^{\mathrm{IF}}}\left(x_{k}\right)\right)+\mu_{A_{i}^{\mathrm{IF}}}\left(x_{j}\right)},
\end{aligned}
$$$$
\tilde{b}_{j}
$$

$$
\begin{aligned}
& =\sum_{A_{i}^{\mathrm{IF}} \in \mathbb{F}} \frac{m\left(A_{i}^{\mathrm{IF}}\right)\left(1-v_{A_{i}^{\mathrm{IF}}}\left(x_{j}\right)\right)}{\sum_{j=1}^{n} \mu A_{i}^{\mathrm{IF}}\left(x_{j}\right)+\pi_{A_{i}^{\mathrm{IF}}}\left(x_{j}\right)} \\
& =\sum_{A_{i}^{\mathrm{IF}} \in \mathbb{F}} \frac{m\left(A_{i}^{\mathrm{IF}}\right)\left(1-v_{A_{i}^{\mathrm{IF}}}\left(x_{j}\right)\right)}{\sum_{k=1, k \neq j}^{n} \mu_{A_{i}^{\mathrm{II}}}\left(x_{k}\right)+\mu_{A_{i}^{\mathrm{IF}}}\left(x_{j}\right)+\pi_{A_{i}^{\mathrm{IF}}}\left(x_{j}\right)} \\
& =\sum_{A_{i}^{\mathrm{IF}} \in \mathbb{F}} \frac{m\left(A_{i}^{\mathrm{IF}}\right)\left(1-v_{A_{i}^{\mathrm{IF}}}\left(x_{j}\right)\right)}{\sum_{k=1, k \neq j}^{n} \mu_{A_{i}^{\mathrm{II}}}\left(x_{k}\right)+\left(1-v_{A_{i}^{\mathrm{II}}}\left(x_{j}\right)\right)} .
\end{aligned}
$$


For, $\forall q \in\{1,2, \ldots, n\}$, we have

$$
\begin{aligned}
& \sum_{\substack{j=1 \\
j \neq q}}^{n} \frac{\mu_{A_{i}^{\mathrm{IF}}}\left(x_{j}\right)}{\sum_{k=1, k \neq j}^{n}\left(1-v_{A_{i}^{\mathrm{IF}}}\left(x_{k}\right)\right)+\mu_{A_{i}^{\mathrm{IF}}}\left(x_{j}\right)} \\
& \leq \sum_{\substack{j=1 \\
j \neq q}}^{n} \frac{\mu_{A_{i}^{\mathrm{IF}}}\left(x_{j}\right)}{\left(1-\mu_{A_{i}^{\mathrm{IF}}}\left(x_{q}\right)\right)+\sum_{k=1, k \neq q}^{n} \mu_{A_{i}^{\mathrm{IF}}}\left(x_{k}\right)} \\
& =\frac{\sum_{j=1, j \neq q}^{n} \mu_{A_{i}^{\mathrm{IF}}}\left(x_{j}\right)}{\left(1-\mu_{A_{i}^{\mathrm{IF}}}\left(x_{q}\right)\right)+\sum_{k=1, k \neq q}^{n} \mu_{A_{i}^{\mathrm{IF}}}\left(x_{k}\right)} \\
& =1-\frac{1-\mu_{A_{i}^{\mathrm{IF}}}\left(x_{q}\right)}{\left(1-\mu_{A_{i}^{\mathrm{IF}}}\left(x_{q}\right)\right)+\sum_{k=1, k \neq q}^{n} \mu_{A_{i}^{\mathrm{IF}}}\left(x_{k}\right)}, \\
& \sum_{\substack{j=1 \\
j \neq q}}^{n} \frac{1-v_{A_{i}^{\mathrm{IF}}}\left(x_{j}\right)}{\sum_{k=1, k \neq j}^{n} \mu_{A_{i}^{\mathrm{IF}}}\left(x_{k}\right)+\left(1-v_{A_{i}^{\mathrm{IF}}}\left(x_{j}\right)\right)} \\
& \geq \sum_{\substack{j=1 \\
j \neq q}}^{n} \frac{1-v_{A_{i}^{\mathrm{IF}}}\left(x_{j}\right)}{\left.\mu_{q}\right)+\sum_{k=1, k \neq q}^{n}\left(1-v_{A_{i}^{\mathrm{IF}}}\left(x_{k}\right)\right)} \\
& =\frac{\sum_{j=1, j \neq q}^{n} 1-v_{A_{i}^{\mathrm{IF}}}\left(x_{j}\right)}{\mu_{A_{i}^{\mathrm{IF}}}\left(x_{q}\right)+\sum_{k=1, k \neq q}^{n}\left(1-v_{A_{i}^{\mathrm{IF}}}\left(x_{k}\right)\right)} \\
& =1-\frac{\mu_{A_{i}^{\mathrm{IF}}}\left(x_{q}\right)}{\mu_{A_{i}^{\mathrm{IF}}}\left(x_{q}\right)+\sum_{k=1, k \neq q}^{n}\left(1-v_{A_{i}^{\mathrm{IF}}}\left(x_{k}\right)\right)} \text {. }
\end{aligned}
$$

We can also obtain

$$
\begin{aligned}
& \sum_{\substack{j=1 \\
j \neq q}}^{n} \widetilde{a}_{q}=\sum_{\substack{j=1 \\
j \neq q}}^{n} \sum_{A_{i}^{\mathrm{IF}} \in \mathbb{F}} \frac{m\left(A_{i}^{\mathrm{IF}}\right) \mu_{A_{i}^{\mathrm{IF}}}\left(x_{j}\right)}{\sum_{k=1, k \neq j}^{n}\left(1-v_{A_{i}^{\mathrm{IF}}}\left(x_{k}\right)\right)+\mu_{A_{i}^{\mathrm{IF}}}\left(x_{j}\right)} \\
& =\sum_{A_{i}^{\mathrm{IF}} \in \mathbb{F}}\left(m\left(A_{i}^{\mathrm{IF}}\right)\right. \\
& \left.\cdot \sum_{\substack{j=1 \\
j \neq q}}^{n} \frac{\mu_{A_{i}^{\mathrm{IF}}}\left(x_{j}\right)}{\sum_{k=1, k \neq j}^{n}\left(1-v_{A_{i}^{\mathrm{IF}}}\left(x_{k}\right)\right)+\mu_{A_{i}^{\mathrm{IF}}}\left(x_{j}\right)}\right) \\
& \leq \sum_{A_{i}^{\mathrm{IF}} \in \mathbb{F}}\left(m\left(A_{i}^{\mathrm{IF}}\right)\right. \\
& \left.\cdot\left(1-\frac{1-v_{A_{i}^{\mathrm{IF}}}\left(x_{k}\right)}{1-v_{A_{i}^{\mathrm{IF}}}\left(x_{q}\right)+\sum_{k=1, k \neq q}^{n} \mu_{A_{i}^{\mathrm{IF}}}\left(x_{k}\right)}\right)\right) \\
& =\sum_{A_{i}^{\mathrm{IF}} \in \mathbb{F}} m\left(A_{i}^{\mathrm{IF}}\right) \\
& -\sum_{A_{i}^{\mathrm{IF}} \in \mathbb{F}} \frac{m\left(A_{i}^{\mathrm{IF}}\right)\left(1-v_{A_{i}^{\mathrm{IF}}}\left(x_{k}\right)\right)}{1-v_{A_{i}^{\mathrm{IF}}}\left(x_{q}\right)+\sum_{k=1, k \neq q}^{n} \mu_{A_{i}^{\mathrm{IF}}}\left(x_{k}\right)}=1-\tilde{b}_{q},
\end{aligned}
$$

$$
\begin{aligned}
& \sum_{\substack{j=1 \\
j \neq q}}^{n} \widetilde{b}_{q}=\sum_{\substack{j=1 \\
j \neq q}}^{n} \sum_{A_{i}^{\mathrm{I}} \in \mathbb{F}} \frac{m\left(A_{i}^{\mathrm{IF}}\right)\left(1-v_{A_{i}^{\mathrm{IF}}}\left(x_{j}\right)\right)}{\sum_{k=1, k \neq j}^{n} \mu_{A_{i}^{\mathrm{IF}}}\left(x_{k}\right)+\left(1-v_{A_{i}^{\mathrm{IF}}}\left(x_{j}\right)\right)} \\
& =\sum_{A_{i}^{\mathrm{IF}} \in \mathbb{F}}\left(m\left(A_{i}^{\mathrm{IF}}\right)\right. \\
& \left.\cdot \sum_{\substack{j=1 \\
j \neq q}}^{n} \frac{1-v_{A_{i}^{\mathrm{IF}}}\left(x_{j}\right)}{\sum_{k=1, k \neq j}^{n} \mu_{A_{i}^{\mathrm{IF}}}\left(x_{k}\right)+\left(1-v_{A_{i}^{\mathrm{IF}}}\left(x_{j}\right)\right)}\right) \\
& \geq \sum_{A_{i}^{\mathrm{IF}} \in \mathbb{F}}\left(m\left(A_{i}^{\mathrm{IF}}\right)\right. \\
& \left.\cdot\left(1-\frac{\mu_{A_{i}^{\mathrm{IF}}}\left(x_{q}\right)}{\mu_{A_{i}^{\mathrm{IF}}}\left(x_{q}\right)+\sum_{k=1, k \neq q}^{n}\left(1-v_{A_{i}^{\mathrm{IF}}}\left(x_{k}\right)\right)}\right)\right) \\
& =\sum_{A_{i}^{\mathrm{IF}} \in \mathbb{F}} m\left(A_{i}^{\mathrm{IF}}\right) \\
& -\sum_{A_{i}^{\mathrm{IF}} \in \mathbb{F}} \frac{m\left(A_{i}^{\mathrm{IF}}\right) \mu_{A_{i}^{\mathrm{IF}}}\left(x_{q}\right)}{\mu_{i}^{\mathrm{IF}}\left(x_{q}\right)+\sum_{k=1, k \neq q}^{n}\left(1-v_{A_{i}^{\mathrm{IF}}}\left(x_{k}\right)\right)}=1 \\
& -\widetilde{a}_{q} \text {. }
\end{aligned}
$$

Finally we can get

$$
\begin{aligned}
& \sum_{\substack{j=1 \\
j \neq q}}^{n} \widetilde{a}_{q} \leq 1-\widetilde{b}_{q}, \\
& \sum_{\substack{j=1 \\
j \neq q}}^{n} \widetilde{b}_{q} \geq 1-\widetilde{a}_{q}, \quad \forall q \in\{1,2, \ldots, n\} .
\end{aligned}
$$

Hence

$$
\begin{aligned}
& \sum_{j=1}^{n} \widetilde{a}_{j}+\left(\widetilde{b}_{q}-\widetilde{a}_{q}\right) \leq 1, \\
& \sum_{j=1}^{n} \widetilde{b}_{j}-\left(\widetilde{b}_{q}-\tilde{a}_{q}\right) \geq 1,
\end{aligned}
$$

$\forall q \in\{1,2, \ldots, n\}$.

Therefore, $\widetilde{P}\left(x_{j}\right)=\left[\widetilde{a}_{j}, \widetilde{b}_{j}\right]$ is an interval-valued probability distribution in $X$.

Proposition 22. The probability estimation $\widetilde{P}\left(x_{j}\right)=\left[\widetilde{a}_{j}, \widetilde{b}_{j}\right]$ is identical to $\widetilde{P}\left(x_{j}\right)(j=1,2, \ldots, n)$ if $A_{i}^{I F}$ reduces to a fuzzy set.

Proof. Since $\pi_{A_{i}^{\mathrm{IF}}}\left(x_{j}\right)=0, \forall A_{i}^{\mathrm{IF}} \in \mathbb{F}, j=1,2, \ldots, n$, we have $\mu_{A_{i}^{\mathrm{IF}}}\left(x_{j}\right)=1-v_{A_{i}^{\mathrm{IF}}}\left(x_{j}\right)$. 
Then $\tilde{a}_{j}$ in (26) is identical to $\widetilde{b}_{j}$ in (27); we get

$$
\widetilde{P}\left(x_{j}\right)=\tilde{a}_{j}=\tilde{b}_{j}=\sum_{A_{i}^{\mathrm{IF}} \in \mathbb{F}} \frac{m\left(A_{i}^{\mathrm{IF}}\right) \mu_{A_{i}^{\mathrm{IF}}}\left(x_{j}\right)}{\sum_{j=1}^{n} \mu_{A_{i}^{\mathrm{IF}}}\left(x_{j}\right)} .
$$

So $\widetilde{P}\left(x_{j}\right)=\left[\tilde{a}_{j}, \widetilde{b}_{j}\right]$ is identical to $\widetilde{P}\left(x_{j}\right)(j=1,2, \ldots, n)$.

Considering Proposition 22, we can take the probability estimation in (22) for a special case of interval probability estimation in (25).

Now let us reinvestigate Example 15 by (25)-(27); we can get the probability distribution in $X$ as

$$
\begin{aligned}
& \widetilde{P}(30)=[0.15,0.22], \\
& \widetilde{P}(40)=[0.19,0.27], \\
& \widetilde{P}(50)=[0.24,0.33], \\
& \widetilde{P}(60)=[0.14,0.22], \\
& \widetilde{P}(70)=[0.10,0.16] .
\end{aligned}
$$

The probability distribution can be applied for decision making directly by comparing these intervals. However, the comparison of intervals is challengeable, especially for intervals with overlaps. Many methods for comparison of intervals have been proposed [40-43], most of which are based on the midpoints of interval numbers [44]. For simplicity, we take the method mentioned in [40], where the comparison of intervals was executed by comparing their centers. By this method, we can get $\widetilde{P}(50) \geq \widetilde{P}(40) \geq \widetilde{P}(30) \geq \widetilde{P}(60) \geq \widetilde{P}(70)$. This indicates that $\widetilde{P}(50)$; that is, the probability of "assigning fifty thousands for advertisement" is the largest one. There is no doubt that this result is much more instructive for the decision maker.

Since the crisp focal elements containing more than one element can be represented by fuzzy focal elements, they can also be expressed by IF focal elements. The focal element $A=\left\{x_{1}, x_{2}, x_{4}\right\}$ in the universe $X=\left\{x_{1}, x_{2}, x_{3}, x_{4}, x_{5}\right\}$ given previously can be written as an IF set as $A^{\mathrm{IF}}=$ $\left\{\left\langle x_{1}, 1,0\right\rangle,\left\langle x_{2}, 1,0\right\rangle,\left\langle x_{3}, 0,1\right\rangle,\left\langle x_{4}, 1,0\right\rangle,\left\langle x_{5}, 0,1\right\rangle\right\}$. It can be verified that the probability estimation based on IF belief functions reduces to the pignistic transformation for crisp focal elements. Therefore, belief functions with crisp focal elements and fuzzy focal elements as well as IF focal elements can be transformed to probability distribution in the universe of discourse by the probability estimation defined in Definition 20. In the following discussions, no differentiation among crisp focal sets, fuzzy sets, and intuitionistic fuzzy sets will be made. The superscripts in $A^{F}$ and $A^{\mathrm{IF}}$ will be ignored.

It is worth noticing that the combination of IF belief functions is still an open topic. If we transform IF belief functions to probability distribution on the universe, it can be considered as interval-valued probability distribution or interval-valued Bayesian belief functions [45]. Then they can be combined by the combination rule for intervalvalued belief functions. In this paper, we attempt to use the method proposed in [45] to combine IF belief functions by transforming them into interval-valued Bayesian belief functions. This combination rule is beyond the scope of this paper; hence, it is not presented here. Readers can refer to [45] that may provide the basis of this rule. In the following section, illustrative examples will be employed to show the applicability of our proposed probability estimation method.

\section{Illustrative Examples}

Example 23. Reconsider the situation discussed in Example 13, where the universe of discourse is $X=\{30,40$, $50,60,70\}$. The assessment result from an expert is

$$
\begin{aligned}
& m(A)=0.15, \\
& m(B)=0.2, \\
& m(C)=0.3, \\
& m(D)=0.25, \\
& m(E)=0.1 .
\end{aligned}
$$

$A, B$, and $C$ are three IF events representing the statements "about fifty thousands," "a large amount of money," and "a small amount of money," respectively. $D$ is the fuzzy event "a middle amount of money". $E$ is a crisp event saying "the amount of money is 60 thousands." These focal elements can be expressed as

$$
\begin{aligned}
A= & \{\langle 30,0.2,0.6\rangle,\langle 40,0.5,0.4\rangle,\langle 50,1,0\rangle, \\
& \langle 60,0.5,0.4\rangle,\langle 70,0.2,0.6\rangle\}, \\
B= & \{\langle 30,0,1\rangle,\langle 40,0.2,0.6\rangle,\langle 50,0.5,0.3\rangle, \\
& \langle 60,0.8,0.1\rangle,\langle 70,1,0\rangle\}, \\
C= & \{\langle 30,1,0\rangle,\langle 40,0.8,0.1\rangle,\langle 50,0.3,0.4\rangle, \\
& \langle 60,0.1,0.8\rangle,\langle 70,0,1\rangle\}, \\
D= & \{\langle 30,0.4,0.6\rangle,\langle 40,0.6,0.4\rangle,\langle 50,1,0\rangle, \\
& \langle 60,0.6,0.4\rangle,\langle 70,0.4,0.6\rangle\}, \\
E= & \{\langle 30,0,1\rangle,\langle 40,0,1\rangle,\langle 50,0,1\rangle,\langle 60,1,0\rangle,
\end{aligned}
$$$$
\langle 70,0,1\rangle\} \text {. }
$$

From Definition 20, we can get the interval probability distribution as

$$
\begin{aligned}
& \widetilde{P}(30)=[0.16,0.20], \\
& \widetilde{P}(40)=[0.18,0.24], \\
& \widetilde{P}(50)=[0.21,0.27], \\
& \widetilde{P}(60)=[0.23,0.28], \\
& \widetilde{P}(70)=[0.11,0.14] .
\end{aligned}
$$


Taking a closer examination on the result, we can find that it satisfies the condition of $\sum_{j=1}^{n} b_{j}-\left(b_{k}-a_{k}\right) \geq 1$, $\forall k \in\{1, \ldots, n\}$, and $\sum_{j=1}^{n} a_{j}+\left(b_{k}-a_{k}\right) \leq 1, \forall k \in\{1, \ldots, n\}$. In addition, we can make an obvious decision that assigning sixty thousands for advertisement is acceptable.

Example 24. Reconsider the situation discussed in Example 13, where the universe of discourse is $X=\{30,40$, $50,60,70\}$. The assessment results from three experts are given as

$$
\begin{aligned}
& m_{1}(A)=0.15, \\
& m_{1}(B)=0.2, \\
& m_{1}(C)=0.3, \\
& m_{1}(D)=0.25, \\
& m_{1}(E)=0.1 ; \\
& m_{2}(A)=0.15, \\
& m_{2}(B)=0.3, \\
& m_{2}(C)=0.4, \\
& m_{2}(F)=0.15 ; \\
& m_{3}(A)=0.1, \\
& m_{3}(B)=0.3, \\
& m_{3}(C)=0.5 \\
& m_{3}(G)=0.1
\end{aligned}
$$

The events $A, B, C, D$, and $E$ have identical interpretations as in the last example. $F$ and $G$ representing "about sixty thousands" and "about seventy thousands," respectively, are given as

$$
\begin{aligned}
F= & \{\langle 30,0,0.9\rangle,\langle 40,0.1,0.7\rangle,\langle 50,0.2,0.5\rangle, \\
& \langle 60,1,0\rangle,\langle 70,0.2,0.5\rangle\}, \\
G= & \{\langle 30,0,1\rangle,\langle 40,0.1,0.8\rangle,\langle 50,0.2,0.4\rangle, \\
& \langle 60,0.5,0.1\rangle,\langle 70,1,0\rangle\} .
\end{aligned}
$$

By Definition 20, the probability distribution corresponding to each IF belief function can be obtained as

$$
\begin{aligned}
& \widetilde{P}_{1}(30)=[0.16,0.20], \\
& \widetilde{P}_{1}(40)=[0.18,0.24], \\
& \widetilde{P}_{1}(50)=[0.21,0.27], \\
& \widetilde{P}_{1}(60)=[0.23,0.28],
\end{aligned}
$$

$$
\begin{aligned}
& \widetilde{P}_{1}(70)=[0.11,0.14] ; \\
& \widetilde{P}_{2}(30)=[0.16,0.22], \\
& \widetilde{P}_{2}(40)=[0.18,0.27], \\
& \widetilde{P}_{2}(50)=[0.17,0.28], \\
& \widetilde{P}_{2}(60)=[0.17,0.27], \\
& \widetilde{P}_{2}(70)=[0.12,0.18] ; \\
& \widetilde{P}_{3}(30)=[0.19,0.25], \\
& \widetilde{P}_{3}(40)=[0.20,0.28], \\
& \widetilde{P}_{3}(50)=[0.16,0.27], \\
& \widetilde{P}_{3}(60)=[012,0.21], \\
& \widetilde{P}_{3}(70)=[0.14,0.19] .
\end{aligned}
$$

These interval-valued probabilities can be regarded as the normalized interval-valued Bayesian belief functions as defined in our earlier paper [45]. So they can be combined by the combination rule proposed in this paper. The normalized result is

$$
\begin{aligned}
& \widetilde{P}(30)=[0.15,0.16], \\
& \widetilde{P}(40)=[0.26,0.27], \\
& \widetilde{P}(50)=[0.28,0.30], \\
& \widetilde{P}(60)=[0.23,0.24], \\
& \widetilde{P}(70)=[0.05,0.06] .
\end{aligned}
$$

It can be verified that the final result satisfies the conditions in Definition 17.

These examples illustrate that the probability estimation method is also suitable for both fuzzy belief functions and classical belief functions. By estimating the probability distribution in the universe, we can make decision more conveniently. Moreover, the probability estimation can provide an alternative way for the combination of IF belief functions.

\section{Conclusion}

The evidence theory has been extended to intuitionistic fuzzy environment to deal with imprecise and vague information. Intuitionistic fuzzy belief functions have received considerable interest for its capability of managing uncertainty in information systems. Uncertainty on the events can be expressed by belief functions on IF sets, while a sounder decision cannot be got based on IF belief functions. So, it is necessary to transform IF belief functions to probability distribution in the universe of discourse. In this paper we mainly investigated the probability estimation based on 
IF belief functions. The probability estimations based on fuzzy and IF belief functions, together with their proofs, are presented. It has been proved that the probabilities of basic events estimated form the fuzzy belief functions are precise value, while those from the IF belief functions are intervals. Decision making can be implemented based on the comparison between intervals. Moreover, when all IF belief functions degenerate into classical belief functions, the probability estimation is identical to the pignistic transformation. In such sense, the probability estimation based on IF belief functions can be regarded as the extension of pignistic transformation to the intuitionistic fuzzy environment. In addition, the proposed probability estimation method provides an alternative combination rule for IF belief functions.

\section{Conflict of Interests}

The authors declare that there is no conflict of interests regarding the publication of this paper.

\section{Acknowledgments}

This work is supported by the National Natural Science Foundation of China under Grants 60975026 and 61273275. The authors would also like to send their sincere gratitude to the editors and anonymous reviewers.

\section{References}

[1] A. P. Dempster, "Upper and lower probabilities induced by a multivalued mapping," Annals of Mathematical Statistics, vol. 38, pp. 325-339, 1967.

[2] G. Shafer, A Mathematical Theory of Evidence, Princeton University Press, Princeton, NJ, USA, 1976.

[3] M. Beynon, D. Cosker, and D. Marshall, "An expert system for multi-criteria decision making using Dempster-Shafer theory," Expert Systems with Applications, vol. 20, no. 4, pp. 357-367, 2001.

[4] S. Benferhat, A. Saffiotti, and P. Smets, "Belief functions and default reasoning," Artificial Intelligence, vol. 122, no. 1-2, pp. 169, 2000.

[5] R. W. Jones, A. Lowe, and M. J. Harrison, "A framework for intelligent medical diagnosis using the theory of evidence," Knowledge-Based Systems, vol. 15, no. 1-2, pp. 77-84, 2002.

[6] Z.-G. Liu, Q. Pan, and J. Dezert, "Evidential classifier for imprecise data based on belief functions," Knowledge-Based Systems, vol. 52, pp. 246-257, 2013.

[7] M. Reformat and R. R. Yager, "Building ensemble classifiers using belief functions and OWA operators," Soft Computing, vol. 12, no. 6, pp. 543-558, 2008.

[8] A. Laha, N. R. Pal, and J. Das, "Land cover classification using fuzzy rules and aggregation of contextual information through evidence theory," IEEE Transactions on Geoscience and Remote Sensing, vol. 44, no. 6, pp. 1633-1641, 2006.

[9] T. Denoeux and P. Smets, "Classification using belief functions: relationship between case-based and model-based approaches," IEEE Transactions on Systems, Man, and Cybernetics, Part B: Cybernetics, vol. 36, no. 6, pp. 1395-1406, 2006.
[10] A. Telmoudi and S. Chakhar, "Data fusion application from evidential databases as a support for decision making," Information and Software Technology, vol. 46, no. 8, pp. 547-555, 2004.

[11] W.-Z. Wu, M. Zhang, H.-Z. Li, and J.-S. Mi, "Knowledge reduction in random information systems via Dempster-Shafer theory of evidence," Information Sciences, vol. 174, no. 3-4, pp. 143-164, 2005.

[12] P. Aggarwal, D. Bhatt, V. Devabhaktuni, and P. Bhattacharya, "Dempster Shafer neural network algorithm for land vehicle navigation application," Information Sciences, vol. 253, pp. 2633, 2013.

[13] Z.-G. Su, Y.-F. Wang, and P.-H. Wang, "Parametric regression analysis of imprecise and uncertain data in the fuzzy belief function framework," International Journal of Approximate Reasoning, vol. 54, no. 8, pp. 1217-1242, 2013.

[14] T. Denoeux, "Maximum likelihood estimation from uncertain data in the belief function framework," IEEE Transactions on Knowledge and Data Engineering, vol. 25, no. 1, pp. 119-130, 2013.

[15] L. A. Zadeh, "Fuzzy sets," Information and Control, vol. 8, pp. 338-353, 1965.

[16] K. T. Atanassov, "Intuitionistic fuzzy sets," Fuzzy Sets and Systems, vol. 20, no. 1, pp. 87-96, 1986.

[17] L. A. Zadeh, "Probability measures of fuzzy events," Journal of Mathematical Analysis and Applications, vol. 23, pp. 421-427, 1968.

[18] L. A. Zadeh, "Fuzzy sets and information granularity," in Advances in Fuzzy Set Theory and Applications, pp. 3-18, NorthHolland, Amsterdam, The Netherlands, 1979.

[19] N. D. Singpurwalla and J. M. Booker, "Membership functions and probability measures of fuzzy sets," Journal of the American Statistical Association, vol. 99, no. 467, pp. 867-889, 2004.

[20] M. Ishizuka, K. S. Fu, and J. T. Yao, "Inference procedures under uncertainty for the problem-reduction method," Information Sciences, vol. 28, no. 3, pp. 179-206, 1982.

[21] R. R. Yager, "Generalized probabilities of fuzzy events from fuzzy belief structures," Information Sciences, vol. 28, no. 1, pp. 45-62, 1982.

[22] H. Ogawa and K. S. Fu, "An inexact inference for damage assessment of existing structures," International Journal of ManMachine Studies, vol. 22, no. 3, pp. 295-306, 1985.

[23] J. Yen, "Generalizing the Dempster-Shafer theory to fuzzy sets," IEEE Transactions on Systems, Man, and Cybernetics, vol. 20, no. 3, pp. 559-570, 1990.

[24] H. Zhu and O. Basir, "An adaptive fuzzy evidential nearest neighbor formulation for classifying remote sensing images," IEEE Transactions on Geoscience and Remote Sensing, vol. 43, no. 8, pp. 1874-1889, 2005.

[25] P. Grzegorzewski and E. Mrówka, "Probability of intuitionistic fuzzy events," in Soft Methods in Probability, Statistics and Data Analysis, pp. 105-115, Springer, Heidelberg, Germany, 2002.

[26] B. Riečan, "Representation of probabilities on IFS events," in Soft Methodology and Random Information Systems, pp. 243248, Springer, Heidelberg, Germany, 2004.

[27] B. Riečan, "On a problem of Radko Mesiar: general form of IFprobabilities," Fuzzy Sets and Systems, vol. 157, no. 11, pp. 14851490, 2006.

[28] T. Gerstenkorn and J. Mańko, "A problem of bifuzzy probability of bifuzzy events," BUSEFAL, vol. 76, pp. 41-47, 1998.

[29] T. Gerstenkorn and J. Mańko, "Remarks on the classical probability of bifuzzy events," in Proceedings of the 4th International 
Conference on Computing Anticipatory Systems (CASYS '00), pp. 190-196, HEC, Liège, Belgium, August 2000.

[30] T. Gerstenkorn and J. Mańko, "On a hesitancy margin and a probability of intuitionistic fuzzy events," Notes Intuitionistic Fuzzy Sets, vol. 7, no. 1, pp. 4-9, 2001.

[31] T. Feng, J.-S. Mi, and S.-P. Zhang, "Belief functions on general intuitionistic fuzzy information systems," Information Sciences, vol. 271, pp. 143-158, 2014.

[32] P. Smets, "The combination of evidence in the transferable belief model," IEEE Transactions on Pattern Analysis and Machine Intelligence, vol. 12, no. 5, pp. 447-458, 1990.

[33] R. R. Yager, "Dempster-Shafer belief structures with interval valued focal weights," International Journal of Intelligent Systems, vol. 16, no. 4, pp. 497-512, 2001.

[34] P. Smets and R. Kennes, "The transferable belief model," Artificial Intelligence, vol. 66, no. 2, pp. 191-234, 1994.

[35] K. T. Atanassov, "New operations defined over the intuitionistic fuzzy sets," Fuzzy Sets and Systems, vol. 61, no. 2, pp. 137-142, 1994.

[36] D. H. Hong and C.-H. Choi, "Multicriteria fuzzy decisionmaking problems based on vague set theory," Fuzzy Sets and Systems, vol. 114, no. 1, pp. 103-113, 2000.

[37] P. Grzegorzewski, "On some basic concepts in probability of IFevents," Information Sciences, vol. 232, pp. 411-418, 2013.

[38] P. Smets, "Analyzing the combination of conflicting belief functions," Information Fusion, vol. 8, no. 4, pp. 387-412, 2007.

[39] R. R. Yager, "On the normalization of fuzzy belief structures," International Journal of Approximate Reasoning, vol. 14, no. 2-3, pp. 127-153, 1996.

[40] V. I. Levin, "Comparison of interval numbers and optimization of interval-parameter systems," Automation and Remote Control, vol. 65, no. 4, pp. 625-633, 2004.

[41] L. Dymova and P. Sevastjanov, "An interpretation of intuitionistic fuzzy sets in terms of evidence theory: decision making aspect," Knowledge-Based Systems, vol. 23, no. 8, pp. 772-782, 2010.

[42] X. Wang and E. E. Kerre, "Reasonable properties for the ordering of fuzzy quantities (II)," Fuzzy Sets and Systems, vol. 118, no. 3, pp. 387-405, 2001.

[43] P. Sevastianov, "Numerical methods for interval and fuzzy number comparison based on the probabilistic approach and Dempster-Shafer theory," Information Sciences, vol. 177, no. 21, pp. 4645-4661, 2007.

[44] Y.-M. Wang, J.-B. Yang, and D.-L. Xu, "A preference aggregation method through the estimation of utility intervals," Computers and Operations Research, vol. 32, no. 8, pp. 2027-2049, 2005.

[45] Y. Song, X. Wang, L. Lei, and A. Xue, "Combination of interval-valued belief structures based on intuitionistic fuzzy set," Knowledge-Based Systems, vol. 67, pp. 61-70, 2014. 


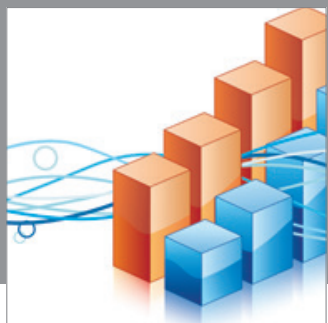

Advances in

Operations Research

mansans

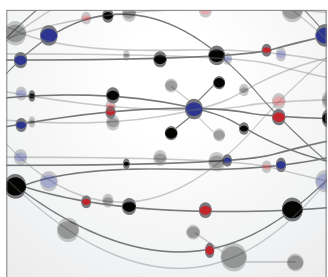

The Scientific World Journal

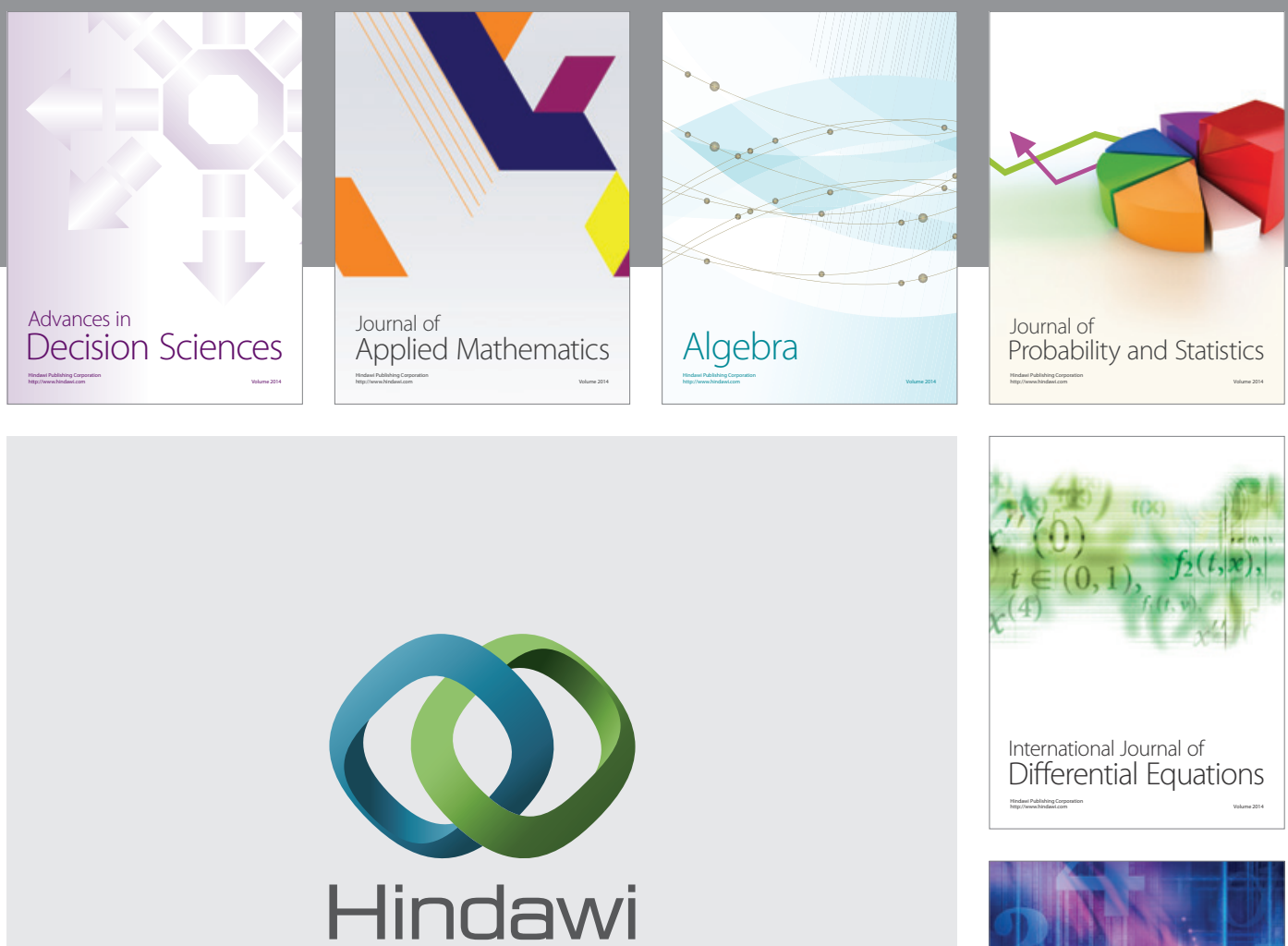

Submit your manuscripts at http://www.hindawi.com
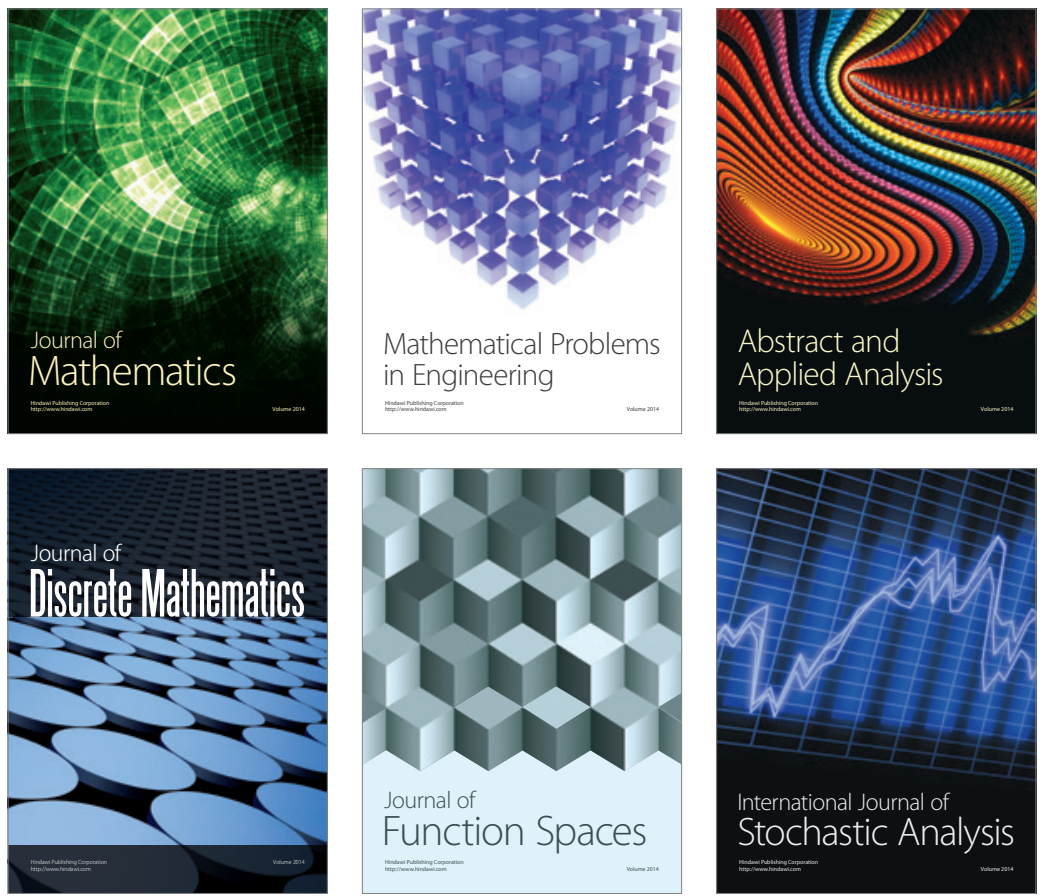

Journal of

Function Spaces

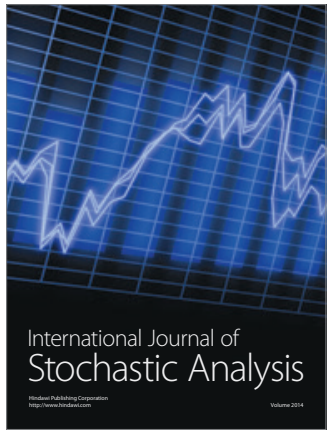

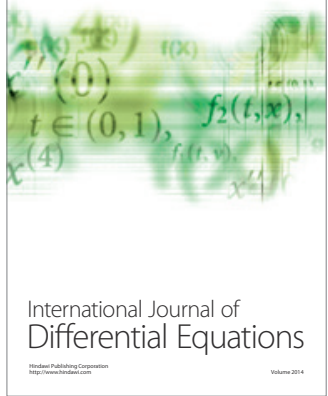
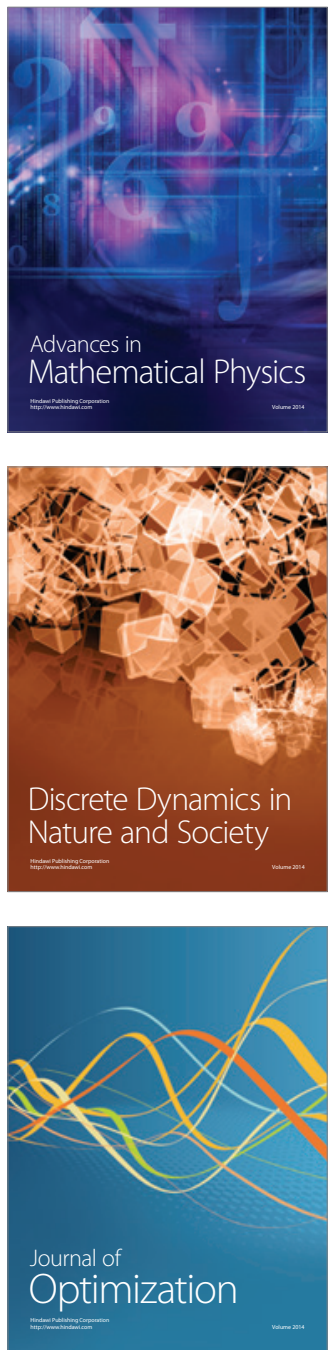